\title{
Acompanhamento da hidratação de cimento Portland simples com resíduo de bauxita
}

\section{(Monitoring hydration of ordinary Portland cement with bauxite residue)}

\author{
R. C.O. Romano*, A. L. Fujii, R. B. Souza, M. S. Takeashi, R. G. Pileggi, M. A. Cincotto \\ Escola Politécnica, Universidade de S. Paulo, Departamento de Engenharia Civil, Av. Prof. Almeida Prado, \\ Trav. 2, 83, S. Paulo, SP 05424-970 \\ *rcorjau@gmail.com
}

\begin{abstract}
Resumo
A substituição parcial de cimento Portland por resíduo de bauxita $(\mathrm{RB})$ resulta em produtos com, no mínimo, propriedades similares às dos produtos fabricados com o ligante puro. No entanto, as interações físico-químicas entre os diferentes tipos de cimento e o RB durante a reação de hidratação ainda é pouco explorada em literatura. A elevada quantidade de sódio e a presença de $\mathrm{Al}_{2} \mathrm{O}_{3}, \mathrm{SiO}_{2} \mathrm{e}$ $\mathrm{Fe}_{2} \mathrm{O}_{3}$ são fatores que afetam a formação dos produtos hidratados e dependem do tipo de cimento utilizado na mistura. Sendo assim, este trabalho foi realizado com o objetivo de avaliar o impacto da presença do resíduo de bauxita em associação ao cimento Portland simples nas primeiras idades de hidratação. Calorimetria de condução isotérmica, difração de raios X, análise termogravimétrica e espectroscopia de infravermelho foram as técnicas escolhidas para o monitoramento. Os resultados ilustraram que a presença do resíduo coletado na planta da Alcoa (Poços de Caldas) aumenta o tempo de indução, o consumo de portlandita, e as quantidades de etringita e monossulfoaluminato formadas. Ao mesmo tempo, houve formação de silicoaluminato de sódio e bicarbonato de sódio, devido às reações com silicatos, aluminatos e com o carbonato do cimento.
\end{abstract}

Palavras-chave: resíduo de bauxita, cimento, hidratação, termogravimetria, DRX, infravermelho.

\begin{abstract}
The partial substitution of Portland cement by bauxite residue (BR) results in products with, at least, properties similar of products with pure binder. However, the physicochemical interactions between Portland cement and BR during the chemical hydration reaction is still poorly explored in literature. The high amount of sodium and presence of $\mathrm{Al}_{2} \mathrm{O}_{3}, \mathrm{SiO}_{2}$ and $\mathrm{Fe}_{2} \mathrm{O}_{3}$ are factors that affect the formation of hydrated products and depends on the type of Portland cement used. The main purpose of this work was to evaluate the impact of use bauxite residue in substitution of part of cement on the early age of hydration. Isothermal conduction calorimetry, X-ray diffraction, thermogravimetry and FTIR were the methods to this monitoring. The results show that the presence of BR, from Alcoa (Poços de Caldas, Brazil), increases the induction period, the portlandite consumption and the amount of ettringite and monosulfoaluminate produced. At the same time, there are the formation of sodium silicoaluminate hydrate and sodium bicarbonate, due to the reactions with silicates, aluminates and with carbonate from cement.
\end{abstract}

Keywords: bauxite residue, cement, hydration, thermogravimetry, X-ray diffraction, FTIR.

\section{INTRODUÇÃO}

O potencial de uso do resíduo de bauxita (RB) como material cimentício suplementar (MCS) está sendo cada vez mais investigado pois, até o momento, trata-se de um rejeito sem aplicação em larga escala embora apresente propriedades físicas, químicas e mineralógicas com bom sinergismo com o ligante hidráulico [1]. Alguns trabalhos já foram conduzidos nesta direção, por exemplo, na tentativa de obtenção de cimentos especiais empregando calcário, resíduo de bauxita, cinza volante, bauxita e gipsita $[2,3]$. Outros foram direcionados para a utilização do RB na fabricação de clínquer Portland, alternativa mais comum atualmente [4-6]. Porém, essa forma de utilização, além de permitir a aplicação de, no máximo, 3\% do resíduo, não diminui o impacto ambiental da cadeia de produção de cimento, pois não reduz as emissões de $\mathrm{CO}_{2}$ para a atmosfera. Foi comprovado que é possível a substituição parcial de cimento Portland por até $20 \%$ do resíduo coletado na Alcoa - Poços de Caldas, resultando em aumento na resistência mecânica e diminuição da permeabilidade ao ar [7]. No entanto, os resultados não podem ser extrapolados para resíduos de bauxita provenientes de outras plantas de obtenção de alumínio, visto que as características físicoquímicas são distintas, resultando em interações diferentes com os cimentos.

A grande maioria dos trabalhos encontrados em literatura tem como foco principal a avaliação dos materiais cimentícios no estado endurecido, com poucas pesquisas sendo realizadas com ênfase na avaliação das propriedades no estado fresco, seja a partir da caracterização reológica ou no acompanhamento das reações químicas do cimento 
[1]. Por se tratar de um material que, devido à composição química e mineralógica, interage com o cimento, alterando a formação dos compostos hidratados e a microestrutura, o monitoramento das alterações que ocorrem durante a consolidação da pasta e o endurecimento ao longo do tempo são de primordial importância. Assim, o foco principal deste trabalho foi de avaliar as interações entre o resíduo de bauxita e o cimento Portland simples nas primeiras idades de hidratação.

\section{PROCEDIMENTO EXPERIMENTAL}

Materiais: o trabalho foi realizado com cimento Portland simples (CPI) e resíduo de bauxita (RB) coletado na planta de produção de alumina da Alcoa América Latina, localizada em Poços de Caldas, região sudeste do Brasil. O RB foi coletado em um teste para avaliação do potencial de aplicação de um filtro prensa, recebido em pellets, sendo necessária uma prévia preparação para a utilização em substituição parcial ao cimento: moagem em moinho de facas, coleta nas mangas e peneiramento em peneira com abertura de malha de $106 \mu \mathrm{m}$.

Mistura das pastas: foram avaliadas uma composição formulada com cimento Portland simples (CPI) e outra com substituição parcial de $10 \%$ do ligante por resíduo de bauxita. A relação água/sólidos foi mantida constante em 0,45 e a mistura preparada em um misturador de bancada com hélice naval (Labortechnik RW20, Ika). Todo o pó foi colocado no béquer e toda a água adicionada, aguardando-se 1 min até a molhagem das partículas. A agitação mecânica foi iniciada com velocidade de rotação de $1500 \mathrm{rpm}$ e mantida por 2 min. Em seguida, as suspensões foram distribuídas em copos plásticos e mantidas a $23 \pm 2{ }^{\circ} \mathrm{C}$ e umidade relativa de $50 \% \pm 2 \%$ para a cura nos tempos pré-definidos.

Preparo das amostras: após $30 \mathrm{~min}, 1,2,4,8,24$ e 48 h, a hidratação do cimento foi interrompida imergindo-se cada amostra em nitrogênio líquido por $2 \mathrm{~min}$. Após o congelamento, as amostras foram acondicionadas em freezer em temperatura inferior a $-25^{\circ} \mathrm{C}$, para evitar qualquer reação e, em seguida, liofilizadas por $24 \mathrm{~h}$ em um equipamento da Terroni Fauvel, LC1500. Nesta etapa, as águas livre, adsorvida e parte da interlamelar congelada na estrutura da pasta foram retiradas por sublimação. Após a liofilização, as amostras foram colocadas em dessecador com sílica gel e mantidas sob vácuo até o momento de cada ensaio. Para a realização dos ensaios descritos a seguir, as amostras foram moídas, em almofariz e pistilo de ágata, e peneiradas em peneira de abertura de malha de $75 \mu \mathrm{m}$, utilizando-se o material passante coletado no fundo da peneira.

Métodos de ensaio. (i) Calorimetria de condução isotérmica: realizada em um equipamento TAM Air (TA Instruments) com precisão de $\pm 20 \mathrm{~mW}$. A temperatura foi mantida em $23{ }^{\circ} \mathrm{C}$ por $48 \mathrm{~h}$. O cimento ou a mistura (cimento $+\mathrm{RB}$ ) foram pesados em uma ampola e a água adicionada com o auxílio de uma seringa. A ampola foi vedada e a suspensão misturada por $30 \mathrm{~s}$. Todos os ensaios tiveram início com 1,5 min após a adição da água no pó seco. (ii)
Difração de raios X (DRX): realizada em um equipamento Empyrean, Panalytical, com detector PIXcel ${ }^{3 \mathrm{D}}$. Os ensaios foram realizados com radiação de cobre, utilizando uma fenda automática de $0,5^{\circ}$, filtro de níquel e frequência de spinning de $2 \mathrm{~s}$ por rotação. As medidas foram feitas com passo de $0,013^{\circ}$, permanecendo em cada passo por $60 \mathrm{~s}$, no intervalo de $5^{\circ}<2 \theta<70^{\circ}$. (iii) Análise termogravimétrica: foi realizada em uma termobalança Netzsch, STA 409 PG, com controle da taxa de aquecimento em $10{ }^{\circ} \mathrm{C} / \mathrm{min}$ até $1000^{\circ} \mathrm{C}$, em atmosfera de $\mathrm{N}_{2}$. A quantidade de pó foi padronizada em $0,8 \mathrm{~g}$ com a finalidade de reduzir as distorções decorrentes de variação da massa inicial; a determinação foi feita em cadinho de alumina com volume de $3,4 \mathrm{~mL}$, sem tampa, sob fluxo de gás $\mathrm{N}_{2} 5.0$ analítico de $20 \mathrm{~mL} / \mathrm{min}$ e os gases volatilizados purgados a uma taxa de $60 \mathrm{~mL} / \mathrm{min}$. (iv) Espectroscopia de infravermelho: a determinação da composição superficial das amostras foi realizada em um equipamento Nicolet (Thermo Scientific), Nexus 6700.

\section{RESULTADOS E DISCUSSÃO}

\section{Caracterização das matérias-primas}

As matérias-primas utilizadas no trabalho apresentam a composição química, determinada por via úmida, conforme descrito na Tabela I, e composição mineralógica, quantificada por difração de raios X, conforme apresentado na Fig. 1 . Adicionalmente, é apresentada a constituição do cimento obtida a partir da aplicação do refinamento de Rietveld, com diferença entre o difratograma obtido e o modelado, menor que $4 \%$. Pelo método de análise empregado, o RB apresenta uma fração solúvel de quase $8,8 \%$, constituída de elevada quantidade de $\mathrm{Na}_{2} \mathrm{O}$ proveniente da digestão do minério com soda cáustica pelo processo Bayer, resultando em um equivalente alcalino elevado de $10,3 \%$. Os elevados teores de $\mathrm{SiO}_{2}$ e $\mathrm{Al}_{2} \mathrm{O}_{3}$ solúveis no resíduo pode contribuir com a formação dos compostos hidratados e ganho de resistência.

Tabela I - Composição química (\% em massa) das matériasprimas obtidas por análise química via úmida.

\section{[Table I - Chemical composition (wt\%) of raw materials.]}

\begin{tabular}{ccc}
\hline Constituinte & CPI & Resíduo de bauxita \\
\hline $\mathrm{CaO}$ & 65,5 & 3,83 \\
$\mathrm{SiO}_{2}$ & 19,7 & $\mathbf{2 0 , 2}$ \\
$\mathrm{Al}_{2} \mathrm{O}_{3}$ & 5,3 & $\mathbf{2 5 , 0}$ \\
$\mathrm{Fe}_{2} \mathrm{O}_{3}$ & 3,4 & 22,6 \\
$\mathrm{SO}_{3}$ & 2,23 & nd \\
$\mathrm{TiO}_{2}$ & nd & 3,37 \\
$\mathrm{Na}_{2} \mathrm{O}$ & $<0,1$ & $\mathbf{8 , 7 8}$ \\
$\mathrm{K}_{2} \mathrm{O}$ & 0,64 & 2,26 \\
Eq. Alc. & 0,45 & 10,3 \\
$\mathrm{PF}$ & 2,16 & 14,1 \\
\hline
\end{tabular}

nd - não determinado. 


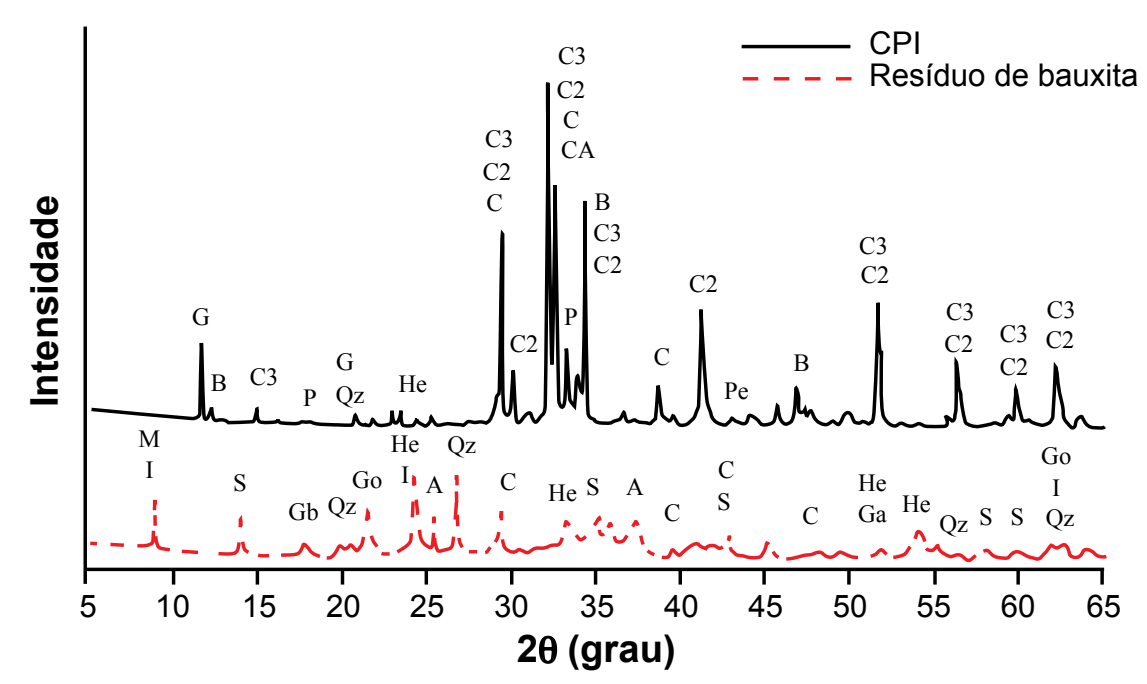

\begin{tabular}{|c|c|c|c|}
\hline Mineral & Fórmula molecular & Representação & $\begin{array}{c}\text { Proporção da fase } \\
\text { no cimento }(\%)\end{array}$ \\
\hline Alita & $3 \mathrm{CaO} \cdot \mathrm{SiO}_{2}$ & $\mathrm{C} 3$ & 64,8 \\
\hline Belita & $2 \mathrm{CaO} \cdot \mathrm{SiO}_{2}$ & $\mathrm{C} 2$ & 16,2 \\
\hline Aluminato tricálcico & $3 \mathrm{CaO} \cdot \mathrm{Al}_{2} \mathrm{O}_{3}$ & $\mathrm{CA}$ & 6,2 \\
\hline Brownmilerita & $4 \mathrm{CaO} \cdot \mathrm{Al}_{2} \mathrm{O}_{3} \cdot \mathrm{Fe}_{2} \mathrm{O}_{3}$ & $\mathrm{~B}$ & 7,2 \\
\hline Calcita & $\mathrm{CaCO}_{3}$ & $\mathrm{C}$ & 0,8 \\
\hline Gipsita & $\mathrm{CaSO}_{4} \cdot 2 \mathrm{H}_{2} \mathrm{O}$ & G & 3,0 \\
\hline Portlandita & $\mathrm{Ca}(\mathrm{OH})_{2}$ & $\mathrm{P}$ & 1,0 \\
\hline Periclásio & $\mathrm{MgO}$ & $\mathrm{Pe}$ & 0,1 \\
\hline Hematita & $\mathrm{Fe}_{2} \mathrm{O}_{3}$ & $\mathrm{He}$ & 0,8 \\
\hline Muscovita & $\mathrm{KAl}_{2}\left(\mathrm{Si}_{3} \mathrm{Al}\right) \mathrm{O}_{10}(\mathrm{OH}, \mathrm{F})_{2}$ & M & \\
\hline Ilita & $\begin{array}{c}\left(\mathrm{K}, \mathrm{H}_{2} \mathrm{O}\right)(\mathrm{Al}, \mathrm{Mg}, \mathrm{Fe})_{2}(\mathrm{Si}, \mathrm{Al})_{4} \mathrm{O}_{10} \\
{\left[(\mathrm{OH})_{2}, \mathrm{H}_{2} \mathrm{O}\right]}\end{array}$ & I & \\
\hline Sodalita & $\mathrm{Na}_{4}\left(\mathrm{SiAlO}_{4}\right)_{3} \mathrm{Cl}$ & S & \\
\hline Gibsita & $\mathrm{Al}(\mathrm{OH})_{3}$ & $\mathrm{~Gb}$ & \\
\hline Goetita & $\mathrm{FeOOH}$ & Go & \\
\hline Anatásio & $\mathrm{TiO}_{2}$ & A & \\
\hline Quartzo & $\mathrm{SiO}_{2}$ & Qz & \\
\hline
\end{tabular}

Figura 1: Composição mineralógica do cimento e do resíduo de bauxita, obtidas por difração de raios X. As proporções das fases do cimento foram estimadas a partir do refinamento de Rietveld.

[Figure 1: Mineralogical composition of cement and bauxite residue, obtained by X-ray diffraction. The proportions of cement phases were estimated by Rietveld refinement.]

Na fração insolúvel o resíduo apresenta fases mineralógicas características da matéria-prima, com maiores intensidades dos picos de muscovita, sodalita, hematita e quartzo. O baixo teor de gibbsita indica a boa digestão do minério. $\mathrm{O}$ cimento apresenta quantidades comuns das fases do clínquer, com predominância de alita, $3 \%$ de gipsita adicionada durante a moagem, e baixos teores de calcita e portlandita, eventualmente formados no armazenamento. Os resultados convergem com os da análise termogravimétrica de 1,3\% de portlandita e $1,0 \%$ de calcita. A presença de $0,8 \%$ de hematita tipicamente pode ser devida à moagem do clínquer com as bolas de ferro metálico. Tal mineral não interferiu no trabalho, pois também é parte da composição do resíduo de bauxita.

\section{Fluxo de calor durante a reação química}

As reações de hidratação do cimento e o fluxo de calor liberado são diretamente relacionados com a composição físico-química do ligante, tipo e quantidade de material cimentício suplementar, aditivo, relação águacimento, tempo de cura, solubilidade do clínquer, etc. [8]. 


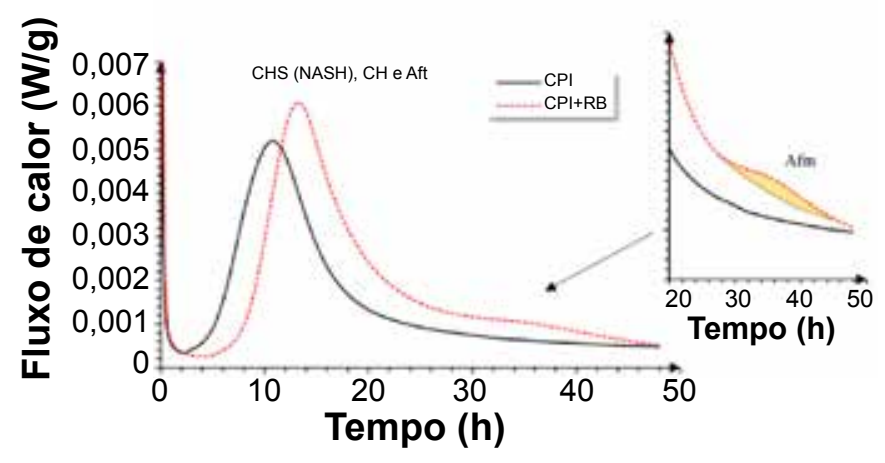

\begin{tabular}{lcc}
\hline Etapa da reação & CPI & CPI+RB \\
\hline Pico de molhagem (W/g) & 0,18 & 0,2 \\
Período de indução (h) & 4,5 & 7,7 \\
Taxa de reação (mW/g/h) & 1,06 & 1,35 \\
Tempo de formação de CH, C-S-H e Aft (h) & 10,8 & 13,2 \\
Calor de formação de CH, C-S-H e Aft (mW/g) & 5,2 & 6,1 \\
Tempo no período de aceleração (h) & 6,3 & 5,5 \\
Calor acumulado após 48 h de reação $(\mathrm{J} / \mathrm{g})$ & 263 & 316 \\
\hline
\end{tabular}

Figura 2: Acompanhamento do fluxo de calor liberado durante a reação química do cimento, com e sem o resíduo de bauxita. Em destaque é apresentada a indicação da inflexão na curva, referente à reação de formação de AFm. Na tabela encontram-se os parâmetros utilizados para a comparação.

[Figure 2: Monitoring the heat release during the cement chemical reaction, with and without bauxite residue. Highlighted is indicated the inflection in the curve, due to the reaction of AFm. In the table parameters used to compare the results are shown.]

Independente desses parâmetros, uma série de reações simultâneas e subsequentes são responsáveis pelo ganho de consistência, ou perda de trabalhabilidade [1]. Desta forma, ocorre a consolidação das suspensões e o consequente ganho de resistência.

Diferentes mecanismos são propostos para descrever a hidratação: tratam-se de reações químicas contínuas e exotérmicas que podem ser divididas em vários estágios, de acordo com o fluxo de calor, conforme já reportado por [9, 10]. No estágio I, que ocorre em minutos, a rápida liberação de calor após o primeiro contato da água com o cimento ocorre devido à dissolução dos álcalis ou sulfato de cálcio e íons, como $\mathrm{K}^{+}, \mathrm{Na}^{+}, \mathrm{SO}_{4}^{2-}, \mathrm{Ca}^{2+}$ [9-12]. No segundo estágio, conhecido como período de indução, as reações de hidratação ocorrem muito vagarosamente, devido à formação de uma camada de gel de C-S-H (silicato de cálcio hidratado) ao redor das partículas de cimento. Essa camada é formada na pasta a partir da agregação de partículas coloidais não estruturadas na superfície do cimento, formando uma camada de somente alguns nanômetros [13]. Este estágio ocorre em um período que vai de minutos até horas, dependendo das características do cimento, adições ou aditivos. Quando a camada de gel é rompida, a difusão iônica é facilitada e a velocidade de reação aumenta. No estágio III, devido à saturação iônica da fase aquosa, ocorre a rápida formação do C-S-H e C-H (hidróxido de cálcio), resultando em redução gradual da concentração de íons de $\mathrm{Ca}^{2+}$. Finalmente, no estágio IV a formação de C-S-H e C-H continuam, mas de forma mais lenta, reduzindo a velocidade da reação global $[9-11,14]$.

O fluxo de calor liberado durante a reação química do cimento, com e sem o resíduo de bauxita, é apresentado na Fig. 2, juntamente com os resultados de cada etapa da reação, para a comparação do impacto da presença do resíduo [9]. A grande quantidade de sódio solúvel e a elevada área superficial específica foram responsáveis pelo aumento no calor liberado na etapa da molhagem. Neste estágio, inicia-se a microcristalização de etringita (AFt), recobrindo os grãos de cimento. Houve aumento no tempo de indução em mais de $3 \mathrm{~h}$, fato creditado à formação de uma camada pouco permeável de C-S-H juntamente com gel de aluminossilicato de sódio hidratado - NASH, conforme já reportado por [15]. Há também alteração da dissolução das fases do clínquer e aumento do consumo de cálcio devido à elevada quantidade de aluminato de sódio $\left[\mathrm{NaAl}(\mathrm{OH})_{4}\right]$, formando aluminato de cálcio hidratado [15]. Esses fatos foram também comprovados a partir do monitoramento da hidratação a partir de difração de raios X.

Apesar do final do período de aceleração das reações ter ocorrido em maior tempo na pasta com resíduo de bauxita $(13,2$ h), a duração desta etapa foi menor, visto que o resíduo acelerou a reação neste período. A elevada alcalinidade do meio favoreceu a precipitação da portlandita e um tempo de formação do C-S-H menor. Esse é um indicativo de que há reatividade entre as fases do clínquer e o material adicionado ao cimento, comprovado também a partir da comparação do calor acumulado após $48 \mathrm{~h}$ de reação. Na pasta com o resíduo de bauxita, em virtude do enriquecimento da solução em aluminato, foi observada uma inflexão com liberação de cerca de $4 \mathrm{~J} / \mathrm{g}$ na curva de calor liberado entre 30 e $40 \mathrm{~h}$ de ensaio em comparação com a reação do cimento puro, referente à conversão de etringita em monossulfoaluminato de cálcio (AFm). Fica muito claro que a utilização do RB afeta a reatividade do cimento, mas somente a partir dos resultados de calorimetria de condução isotérmica não é possível afirmar quais produtos estão sendo formados. Por isso, na sequência são apresentados os resultados do acompanhamento da reação química a partir de difração de raios $\mathrm{X}$, análise termogravimétrica e infravermelho.

\section{Identificação dos compostos hidratados por DRX}

A identificação das fases hidratadas do cimento foi realizada a partir de análise de difração de raios $\mathrm{X}$, após congelamento das amostras em diferentes tempos de hidratação, liofilização, moagem e peneiramento em peneira de abertura de malha $75 \mu \mathrm{m}$. Na Fig. 3 é apresentada uma análise comparativa realizada a partir da contagem em cada pico característico da gipsita, portlandita, etringita e C-S-H. As linhas tracejadas indicam a evolução da formação das fases hidratadas na amostra de cimento e RB, ou consumo, no caso da gipsita, e as linhas contínuas indicam os resultados das pastas com cimento puro. O consumo da gipsita presente na composição dos cimentos é mais acentuado na pasta com 

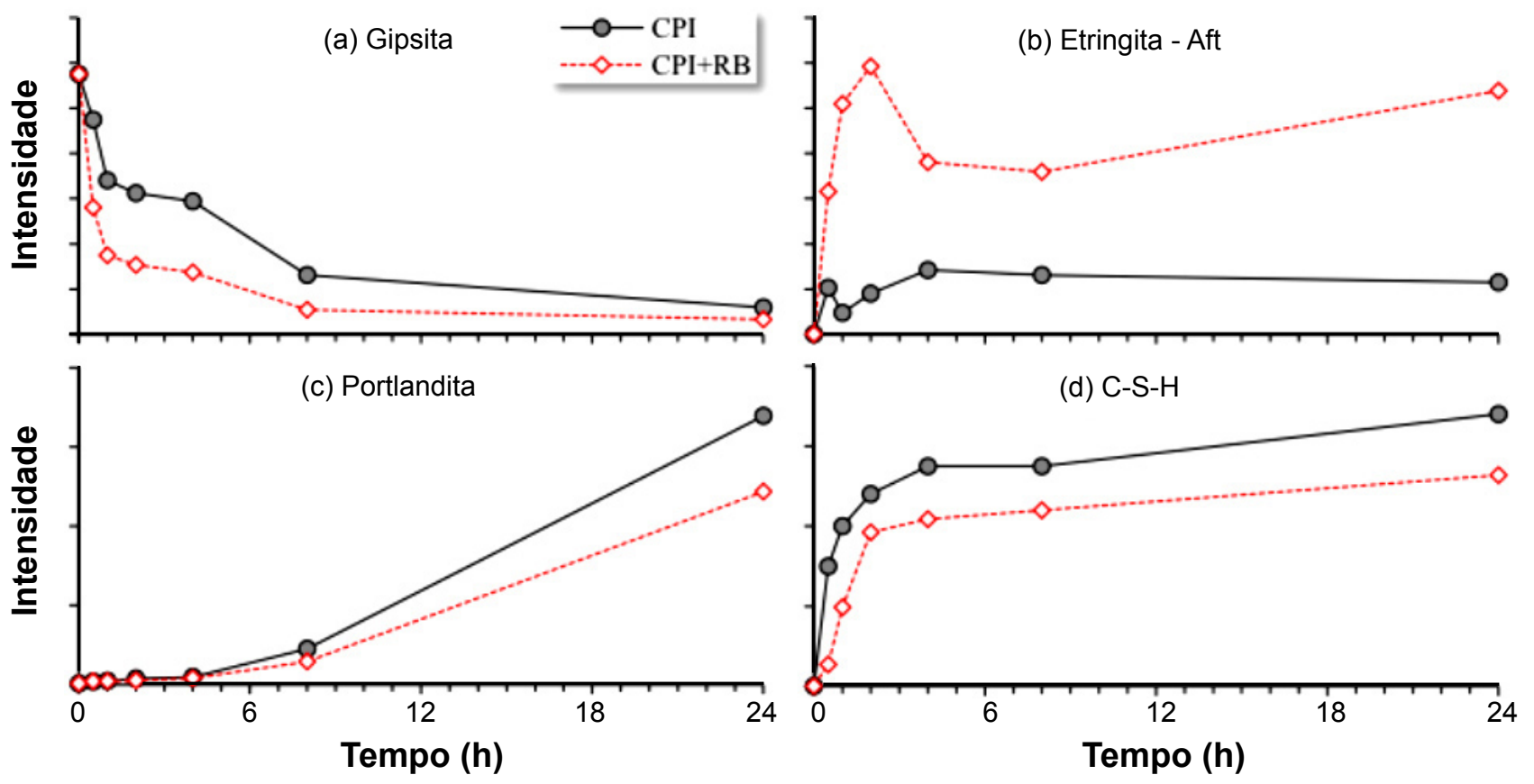

Figura 3: Acompanhamento das alterações mineralógicas durante a hidratação do cimento: consumo de gipsita e formação de portlandita, etringita e C-S-H.

[Figure 3: Monitoring of mineralogical changes during the cement hydration: consumption of gypsum, and formation of portlandite, ettringite and $\mathrm{C}-\mathrm{S}-\mathrm{H}$.
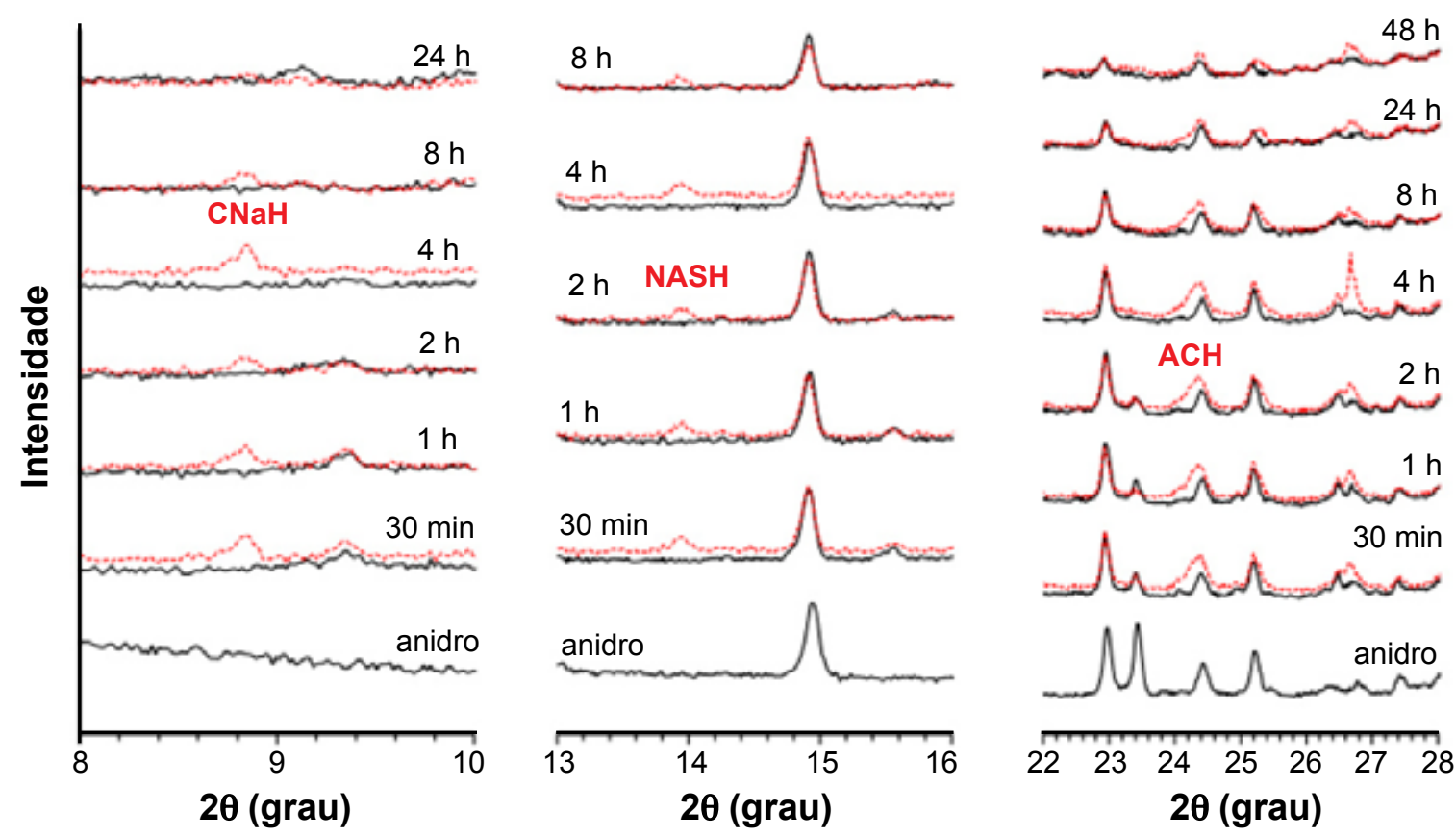

Figura 4: Indicativo da formação de bicarbonato de sódio $(\mathrm{CNaH})$, silicoaluminato de sódio hidratado (NASH) e aluminato de cálcio hidratado $(\mathrm{ACH})$ nos difratogramas de raios $\mathrm{X}$.

[Figure 4: Formation of sodium bicarbonate ( $\mathrm{CNaH})$, sodium silicoaluminate hydrate (NASH), and calcium aluminate hydrate (ACH), in the $X$-ray diffraction patterns.]

o RB devido à reação com o aluminato adicional incorporado com o resíduo. Os picos relacionados à portlandita são menos intensos, indicando que o hidrato está sendo consumido durante a reação, principalmente após $4 \mathrm{~h}$ de hidratação. No entanto, não pode ser dito que há reação pozolânica, pois o resíduo não se enquadra como um material com índice de 
atividade pozolânica de acordo com a normalização técnica.

A formação da etringita foi intensificada em função da presença do RB, em maior teor e em tempo maior, enquanto o C-S-H em menor quantidade, indicando que no período de aceleração das reações (entre 7 e 13 h), o fluxo de calor liberado teve maior impacto em função da formação de AFt. Consequentemente, a conversão em monossulfoaluminato foi intensificada, não sendo possível quantificar tal alteração em função da pobre cristalinidade do hidrato formado. Esse fato foi melhor observado a partir da análise termogravimétrica apresentada mais adiante. A formação de etringita foi superior à do cimento puro e estendeu-se por mais $2 \mathrm{~h}$, diminuindo no período em que se deu a formação do C-S-H, uma vez que ele adsorve o sulfato do meio; enquanto no cimento a formação da etringita é constante, com a adição o seu teor aumenta gradativamente, até $25 \mathrm{~h}$. A evolução de formação do $\mathrm{C}-\mathrm{S}-\mathrm{H}$ segue a mesma cinética, em teor menor.

Apesar dessas informações serem importantes, merece ser discutido isoladamente que, conforme apresentado na Fig. 4, desde o início da hidratação foi observada: i) aceleração da formação do aluminato de cálcio hidratado $(\mathrm{ACH})$, devido à reação dos aluminatos do resíduo com o íon de $\mathrm{Ca}^{2+}$ dissociado liberado pelo cimento; ii) presença de bicarbonato de sódio hidratado nas primeiras $24 \mathrm{~h}$, representado como $\mathrm{CNaH}$, com um pico de maior intensidade em $8,9^{\circ}(2 \theta)$, resultante da reação entre o carbonato de cálcio e o sódio do resíduo em meio fortemente alcalino; e iii) presença de silicoaluminato de sódio hidratado (NASH) nas primeiras $8 \mathrm{~h}$, resultante da reação entre o sódio e as fases silicato e aluminato do clínquer com pico de maior intensidade em $13,9^{\circ}(2 \theta)$. Por isso, menor quantidade de $\mathrm{C}-\mathrm{S}-\mathrm{H}$ foi quantificada, visto que parte dos silicatos solúveis foi utilizada nesta outra reação.

Quantificação das fases decompostas por termogravimetria

O acompanhamento da formação das fases hidratadas a partir de análise termogravimétrica é apresentado na Fig. 5: em (a) estão os resultados da pasta com CPI e em (b) CPI+RB. A quantificação dos compostos hidratados de acordo com os resultados da análise térmica, foi realizada em função da fração volátil avaliada até $1000{ }^{\circ} \mathrm{C}$. Até $350{ }^{\circ} \mathrm{C}$ foi considerada como decomposição dos produtos hidratados dos silicatos, sulfatos, aluminatos e compostos de $\mathrm{Mg}$. Sendo assim, na análise a seguir não foi diferenciada a perda de massa referente à gipsita, $\mathrm{C}-\mathrm{S}-\mathrm{H}$, singenita, etc. A quantidade de portlandita, na maior parte das vezes, foi quantificada entre 360 e $480{ }^{\circ} \mathrm{C}$, porém esta não foi uma faixa fixa para todas as avaliações e dependeu do início e final da decomposição, observados a partir do gráfico

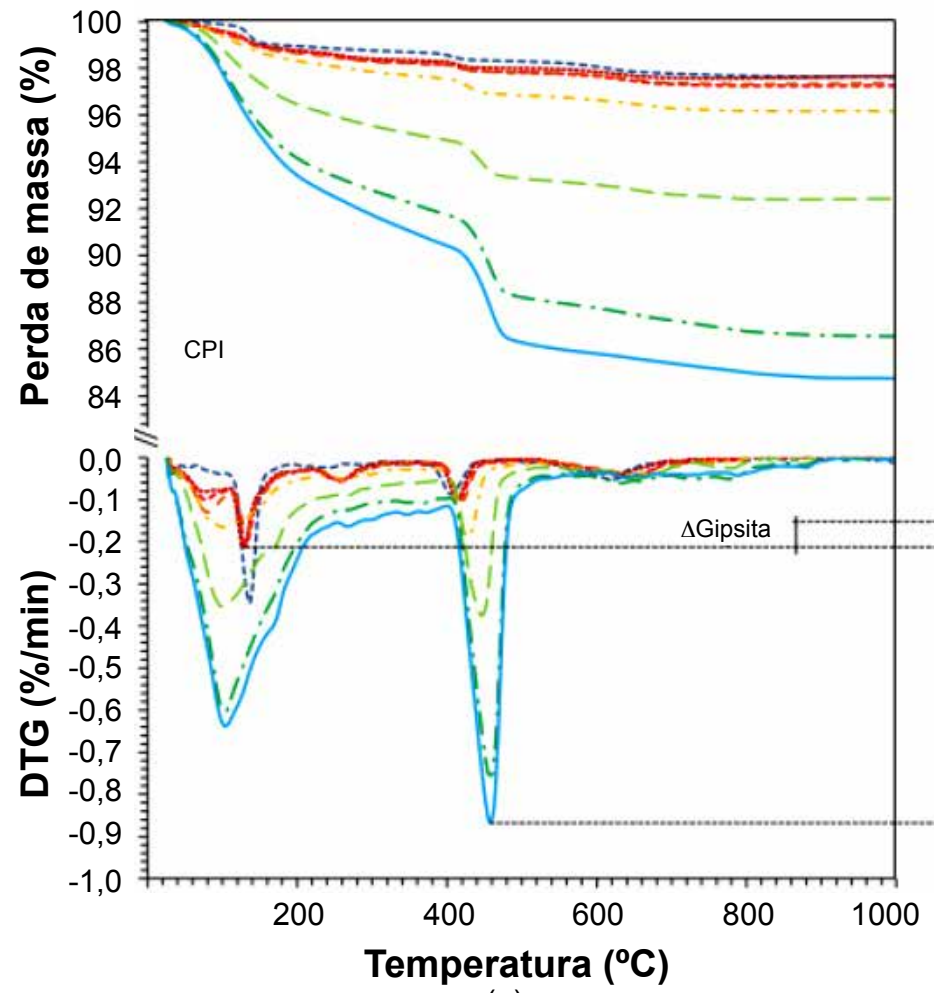

(a)

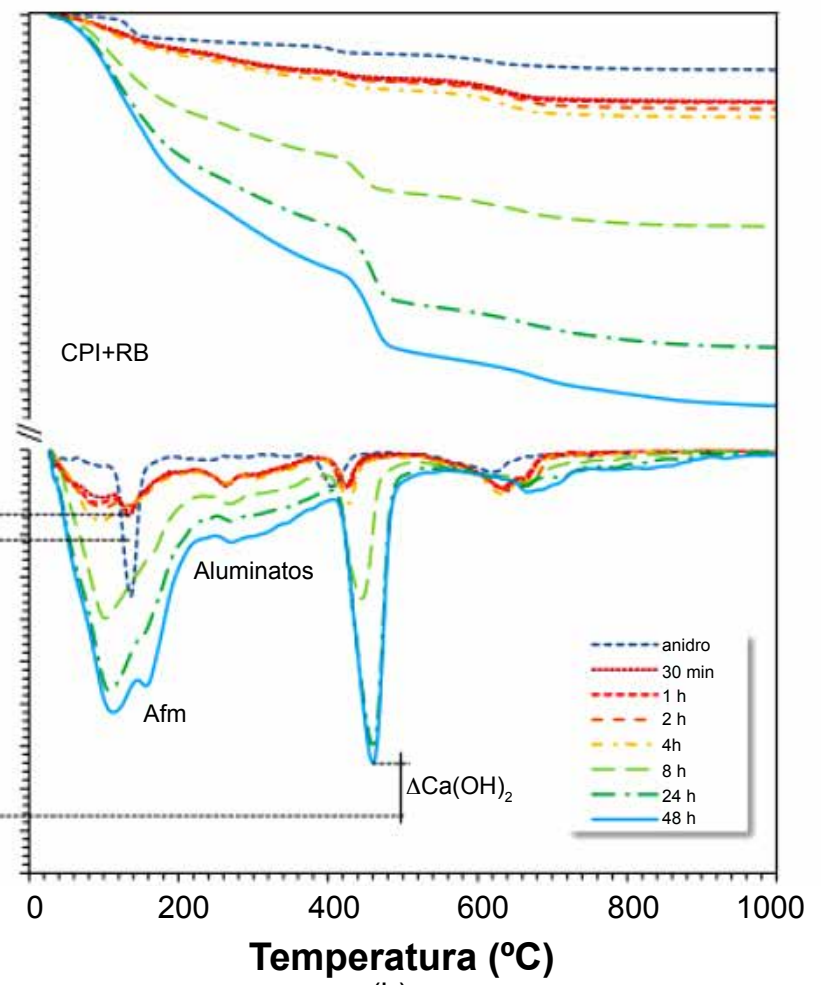

(b)

Figura 5: Decomposição das fases hidratadas. Em (a) estão os resultados para as amostras com o CPI e em (b) das composições de CPI+RB. Em destaque é ilustrada a diferença da quantidade de gipsita, $\mathrm{Ca}(\mathrm{OH})_{2}$ e $\mathrm{AFm}$, em função da utilização do RB.

[Figure 5: Decomposition of hydrated phases. In (a) are the results of CPI and in (b) of CPI+BR. The amount differences of gypsum, $\mathrm{Ca}(\mathrm{OH})_{2}$ and $\mathrm{AFm}$ with addition of bauxite residue are highlighted.] 


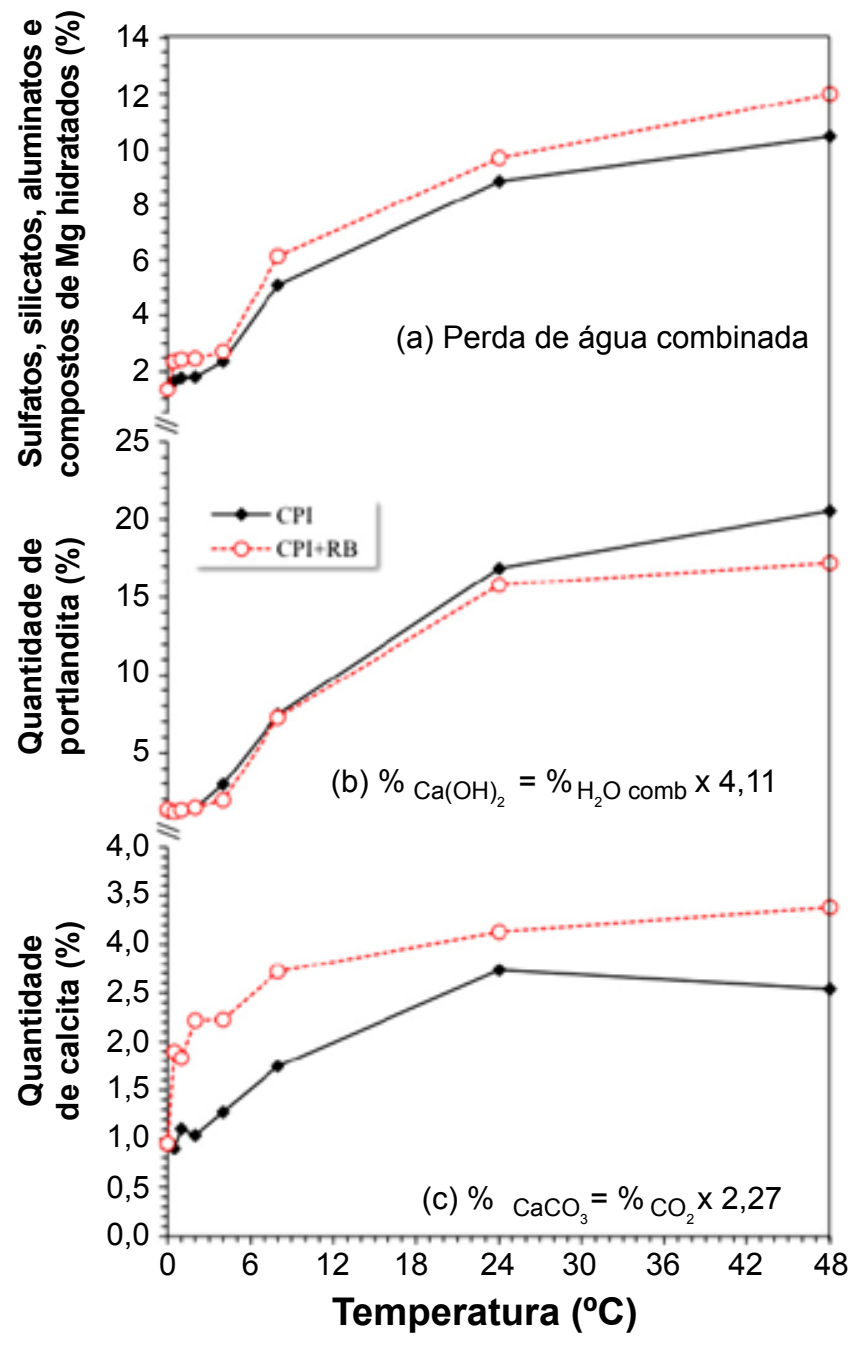

Figura 6: Decomposição da água combinada com os sulfatos, silicatos, aluminatos e compostos de $\mathrm{Mg}$ (a), quantidades de portlandita (b) e de calcita (c).

[Figure 6: Decomposition of water combined as sulfates, silicates, aluminates and compounds of $\mathrm{Mg}(\mathrm{a})$, amount of portlandite (b) and calcite (c).]

da derivada da curva TG. A mesma forma de avaliação foi utilizada para a quantificação da calcita, na faixa de temperatura característica do carbonato $\left(680-800^{\circ} \mathrm{C}\right)$.

A compilação dos resultados obtidos é apresentada na Fig. 6, onde em (a) ilustra-se a perda de água combinada até $350{ }^{\circ} \mathrm{C}$ e em (b) e (c) os teores calculados de portlandita e calcita, respectivamente. Para a portlandita, a porcentagem decomposta foi multiplicada por $4,11\left[\mathrm{MM}_{\mathrm{Ca}(\mathrm{OH})_{2}} / \mathrm{MM}_{\mathrm{H}_{2} \mathrm{O}}\right.$, MM - massa molecular $(\mathrm{g} / \mathrm{mol})]$ e para a calcita o fator de multiplicação foi 2,27 $\left(\mathrm{MM}_{\mathrm{CaCO}_{3}} / \mathrm{MM}_{\mathrm{CO}}\right)$. As porcentagens apresentadas nos gráficos para comparação da perda de massa até $350^{\circ} \mathrm{C}$ equivalem à decomposição bruta, ou seja, não se utilizou nenhum fator de multiplicação, pois a perda de água combinada ocorreu pela decomposição de diferentes fases.

Foi possível visualizar que a utilização do resíduo de bauxita em substituição parcial do cimento intensificou a formação dos compostos hidratados da reação com os silicatos, sulfatos e aluminatos: a formação da etringita foi acelerada, assim como a conversão em monossulfoaluminato, confirmando o que fora observado a partir da calorimetria de condução isotérmica. Entre 100 e $180{ }^{\circ} \mathrm{C}$ ocorre a decomposição da gipsita e, nas amostras com o resíduo de bauxita, ficou nítida a menor perda de massa nesta faixa de temperatura, ilustrando que o consumo do $\mathrm{CaSO}_{4} \cdot 2 \mathrm{H}_{2} \mathrm{O}$ é acelerado em função da presença de maior quantidade dos aluminatos provenientes do RB, convergindo com os resultados da DRX. Na faixa característica da decomposição da portlandita, foi observado aumento crescente do seu teor. Porém, nas composições com o resíduo, menor quantidade foi determinada a partir de $4 \mathrm{~h}$ de hidratação, indicando o consumo ocorrido. Pode-se inferir que a reação preferencial na composição com o RB ocorre com os aluminatos, visto que há indicativo da maior decomposição dos hidratos na faixa de 200 a $350{ }^{\circ} \mathrm{C}$. Apesar do teor de $\mathrm{CaCO}_{3}$ formado ser baixo, houve influência da presença do RB.

\section{Acompanhamento da hidratação por espectroscopia de infravermelho}

O acompanhamento da hidratação do cimento a partir dos espectros obtidos por espectroscopia no infravermelho é apresentado na Fig. 7. Para avaliação do impacto da presença do RB na composição, os resultados foram divididos em três regiões, de acordo com a faixa do número de onda [11, 16-19]: (a) de 4000 a $2700 \mathrm{~cm}^{-1}$ : refere-se principalmente aos estiramentos simétricos $\left(v_{1}\right)$ e assimétricos $\left(v_{3}\right)$ da vibração $\mathrm{O}-\mathrm{H}$ dos sulfatos e/ou hidróxidos, e das bandas referentes ao $\mathrm{CaCO}_{3}$ adicionado ao cimento; (b) de 1700 a $1200 \mathrm{~cm}^{-1}$ : refere-se à deformação fora do plano $\left(v_{2}\right)$ da vibração $\mathrm{O}-\mathrm{H}$ dos sulfatos e/ou etringita, e às bandas do $\mathrm{CaCO}_{3}$ adicionado ao cimento; e (c) de 1200 a $700 \mathrm{~cm}^{-1}$ : ocorrem principalmente devido ao estiramento $v_{1}$ do grupo $\mathrm{CO}_{3}{ }^{2-}$ formado na hidratação, $v_{3}$ do grupo $\mathrm{SO}_{4}{ }^{2-}$ e do $\mathrm{Si}-\mathrm{O}$, deformação $v_{2}$ do $\mathrm{CO}_{3}$, indicação de sílica polimerizada e deformação angular no plano $\left(v_{4}\right)$ do $\mathrm{CO}_{3}{ }^{2-}$ e $\mathrm{SiO}_{4}$.

As tendências observadas foram as mesmas para as pastas com cimento puro ou com substituição parcial do ligante pelo RB, com aumento da intensidade das bandas em função do aumento do tempo de hidratação, devido à formação dos hidratos, carbonatos e polimerização do silicato. As bandas na região entre 3300 e $3700 \mathrm{~cm}^{-1}$ apresentaram considerável aumento de intensidade em função do aumento do tempo de hidratação e da presença do $\mathrm{RB}$, atribuído à vibração do estiramento simétrico $\left(v_{1}\right)$ e/ou assimétrico $\left(v_{2}\right)$ da ligação $\mathrm{O}-\mathrm{H}$ referente à formação de $\mathrm{Ca}(\mathrm{OH})_{2}$, etringita $\mathrm{e}$ monossulfoaluminato. Entre 1300 e $1600 \mathrm{~cm}^{-1}$ há um nítido aumento na intensidade das bandas referentes ao O-H ligado aos sulfatos e etringita, representando a maior quantidade formada em função da presença de RB na composição. Ficou clara a alteração na intensidade da banda na região referente à polimerização da sílica $\left(1010 \mathrm{a} 1080 \mathrm{~cm}^{-1}\right)$, com impacto da presença do RB até $8 \mathrm{~h}$ de hidratação. Consideráveis alterações referentes ao estiramento assimétrico $\left(v_{3}\right)$ do grupo $\mathrm{SO}_{4}^{2-}$, foram observadas entre 1100 e $1200 \mathrm{~cm}^{-1}$, 

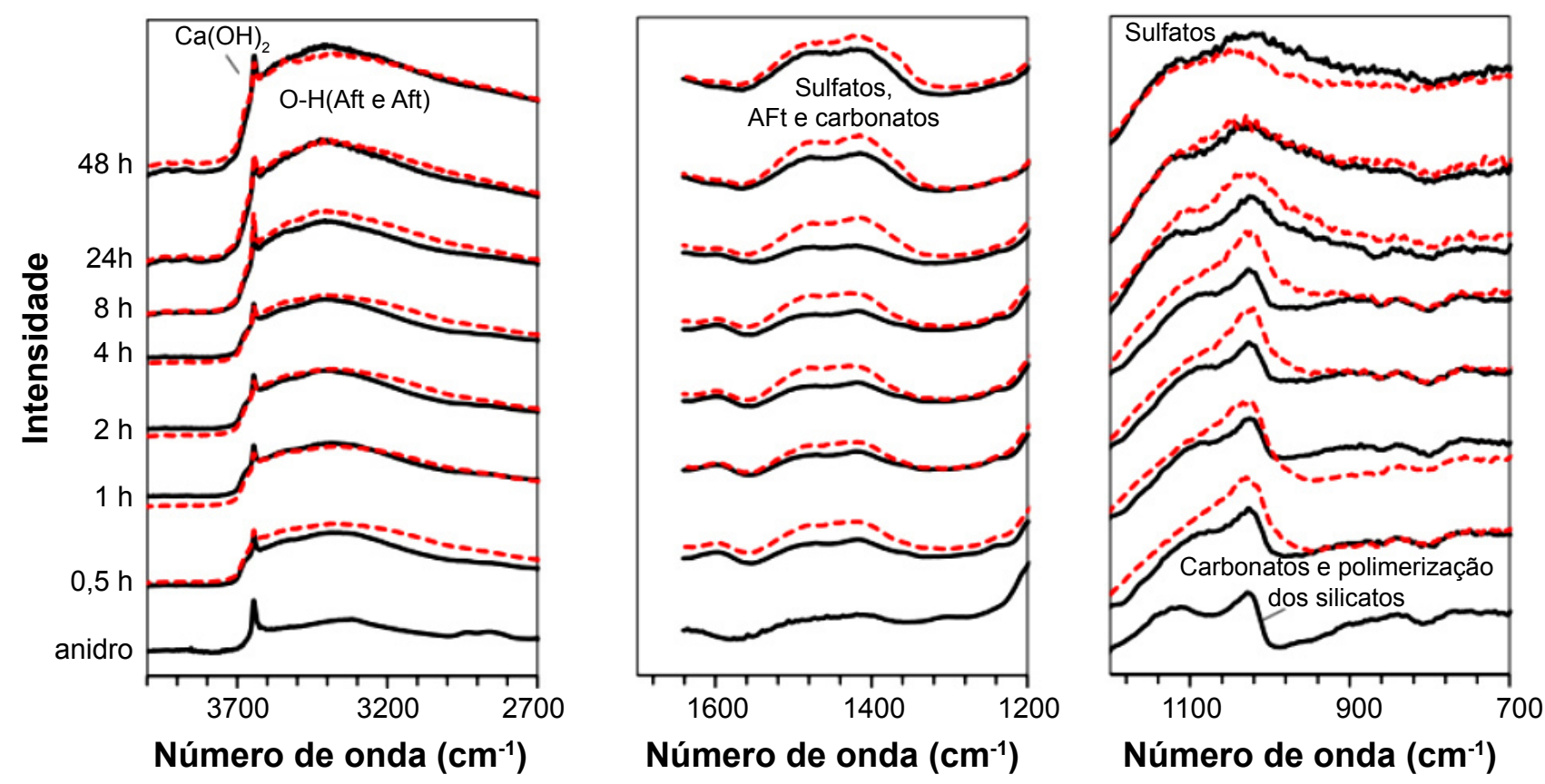

Figura 7: Ampliação das regiões do espectro de infravermelho para ilustração do impacto do uso do resíduo de bauxita na hidratação. [Figure 7: Enlarged IR spectral regions for illustration of the effect of using bauxite residue during hydration reaction.]

com efeito na formação dos compostos hidratados somente até $8 \mathrm{~h}$ de reação. No entanto, deve ser salientado que esta é uma região de difícil avaliação para a hidratação do cimento, visto que vários tipos de sulfato dão origem aos picos que se sobrepõem nesta faixa [18].

A reação mais rápida durante as primeiras horas de hidratação do cimento ocorre devido à formação da etringita. A portlandita formada mais adiante é parcialmente consumida em função das interações com os silicatos para a formação do C-S-H e NASH, conforme indicado por DRX. Em suma, pode-se afirmar que na presença do RB na composição, houve alteração dos picos referentes à: i) formação de maior quantidade de AFt e AFm; ii) reação para formação de carbonatos; iii) aumento do grau de polimerização do silicato. Portanto, apesar de se tratar de um método qualitativo, foi possível identificar as diferenças entre os tempos de hidratação das amostras e entre as amostras formuladas com ou sem o RB durante a reação. Por outro lado, não foi possível a realização de nenhuma observação com relação à quantidade de cada fase formada.

\section{CONCLUSÕES}

O acompanhamento da reação química do cimento em associação ao resíduo de bauxita (RB) é de extrema importância tendo as técnicas complementares aplicadas se mostrado úteis para detalhamento do seu efeito nas primeiras horas de hidratação. Ficou evidente que a presença do RB aumenta o calor liberado durante a reação química, mas retarda o período de indução e o tempo para a formação de $\mathrm{CH}, \mathrm{C}-\mathrm{S}-\mathrm{H}$ (NASH) e AFt [hidróxido de cálcio, silicato de cálcio hidratado (aluminossilicato de sódio hidratado) e etringita, respectivamente], mesmo com aumento na taxa de reação do cimento. A formação da etringita é acelerada e, consequentemente, a transformação em AFm (monossulfoaluminato de cálcio) é intensificada devido à presença do teor elevado de aluminato na composição do RB. Pode-se afirmar que o RB apresenta reatividade com os íons dissociados do cimento durante a hidratação. Com base nos resultados da análise termogravimétrica, foi comprovada a formação de maior quantidade dos hidratos provenientes da reação com os silicatos, sulfatos e aluminatos. $O$ consumo de portlandita na composição com o RB foi nítido a partir de 30 min até $48 \mathrm{~h}$ de hidratação. Dos resultados de infravermelho foi possível observar que a intensificação na formação dos compostos hidratados na composição com o RB ocorre até $24 \mathrm{~h}$ de reação. Foram nítidas as alterações referentes ao estiramento assimétrico $\left(v_{3}\right)$ do grupo $\mathrm{SO}_{4}{ }^{2-} \mathrm{e}$ à vibração do estiramento simétrico $\left(v_{1}\right)$ e/ou assimétrico $\left(v_{2}\right)$ da ligação $\mathrm{O}-\mathrm{H}$, referente à formação de $\mathrm{Ca}(\mathrm{OH})_{2}$, monossulfoaluminato e etringita. A associação de diferentes técnicas para o acompanhamento da hidratação de cimento em associação com o RB foi de extrema importância para a correta compreensão dos fenômenos que governaram a reação química.

\section{AGRADECIMENTOS}

Os autores agradecem à FAPESP, à Alcoa América Latina, ao Instituto de Pesquisas Tecnológicas - IPT e ao Laboratório de Processamento Cerâmico da Engenharia de Materiais na Escola Politécnica da USP, pelo apoio na realização do trabalho. 


\section{REFERÊNCIAS}

[1] R.C.O. Romano, C.C. Liberato, M. Montini, J.B. Gallo, M.A. Cincotto, R.G. Pileggi, "Evaluation of transition from fluid to elastic solid of cementitious pastes with bauxite residue using oscillation rheometry and isothermal calorimetry", App. Rheology 23 (2013) 23830.

[2] M. Singh, S.N. Upadhayay, P.M. Prasad, "Preparation of special cements from red mud", Waste Manage. 16 (1996) 665-670.

[3] M. Singh, S.N. Upadhayay, P.M. Prasad, "Preparation of iron rich cements using red mud", Cem. Concr. Res. 27 (1997) 1037-1046.

[4] L. Alvarez, M. Cabeza, M.A. Climent, J.M. Ortega, X.R. Nóvoa, I. Sanchez, "Microstructure and durability of cements containing red mud", In: XVIII International Congress on the Chemistry of Cement, Madrid (2011).

[5] R.R. Lourenço, J.A. Rodrigues, G.M. Fortes, "Use of bauxite residue as a source of $\mathrm{Al}_{2} \mathrm{O}_{3}$ and $\mathrm{Fe}_{2} \mathrm{O}_{3}$ in the preparation of Portland cement clinker", In: XVIII International Congress on the Chemistry of Cement, Madrid (2011).

[6] A. Talesca, M. Marroccoli, M.L. Pace, G.L. Valenti, "Calcium sulfoaluminate cements obtained from bauxitefree raw mixes", In: XVIII International Congress on the Chemistry of Cement, Madrid (2011).

[7] C.C. Liberato, R.C.O. Romano, J.B. Gallo, J.C. Carvalho, D. Gouvêa, R.G. Pileggi, "Impact of bauxite residue in cement pastes on the hardened state properties", In: Aresty Undergraduate Research Symposium, New Jersey (2011).

[8] Q. Xu, J. Hu, J.M. Ruiz, K. Wang, Z. Ge, "Isothermal calorimetry tests and modeling of cement hydration parameters", Thermochim. Acta 499 (2010) 91-99.

[9] R.C.O. Romano, M.M. Takahashi, C.C. Liberato, R.G. Pileggi, "Fresh and hardened characterization of airentrained cement pastes", In: XIII ICCC International Congress on the Chemistry of Cement, Madrid (2011).
[10] J.S. Lyra, R.C.O. Romano, R.G. Pileggi, D. Gouvêa, "Consolidação de pastas cimentícias contendo policarboxilatos: um estudo calorimétrico e reológico", Cerâmica 58 (2012) 137-143.

[11] H.F.W. Taylor, "Cement Chemistry", Academic Press Ltd., Londres, Inglaterra (1990).

[12] I. Odler, Hydration, setting and hardening of Portland cement, In: P.C. Hewlett Ed., Lea's Chem. Cem. Concr., Elsevier, Oxford (1998).

[13] J.J. Thomas, H.M. Jennings, "A colloidal interpretation of chemical aging of the C-S-H gel and its effects on the properties of cement paste", Cem. Concr. Res. 36 (2006) 3038.

[14] I. Jewed, J. Skalny, J.F. Young, "Hydration of Portland cement”, In: P. Barnes Ed., Struct. Performance Cem. (1983) 237-318.

[15] A.L. Fujji, D.R. Torres, R.C.O. Romano, M.A. Cincotto, R.G. Pileggi, "Impact of superplasticizer on the hardening of slag Portland cement blended with red mud", Construc. Buil. Mater. 101 (2015) 432-439.

[16] T.L. Hughes, C.M. Methven, T.G.J. Jones, S.H. Pelham, P. Fletcher, C. Hall, "Determining cement composition by Fourier transform infrared spectroscopy", Elsevier Sci. Inc., New York, ISSN 1065-7355/95 (1995).

[17] M.A. Trezza, A.E. Lavat, "Analysis of the system $3 \mathrm{CaO}$. $\mathrm{Al}_{2} \mathrm{O}_{3}-\mathrm{CaSO}_{4} \cdot 2 \mathrm{H}_{2} \mathrm{O}-\mathrm{CaCO}_{3}-\mathrm{H}_{2} \mathrm{O}$ by FT-IR spectroscopy", Cem. Concr. Res. 31, 6 (2001) 869-872.

[18] R. Ylmén, U. Jaglid, B.M. Steenari, I. Panas, "Early hydration and setting of Portland cement monitored by IR, SEM and Vicat techniques", Cem. Concr. Res. 39 (2009) 433-439.

[19] E.J. Silva, S.P. Galvão, J.M.F. Mota, R.M.S. Maior, "Análise microestrutural de pastas de cimento Portland branco modificado por um polímero de base acrílica como ferramenta para estudo do comportamento de resistência mecânica", In: Anais do $54^{\circ}$ Congresso Brasileiro do Concreto, Maceió, $\mathrm{Al}$ (2012). (Rec. 19/01/2016, Rev. 13/02/2016, Ac. 13/02/2016) 